\title{
Revista Colombiana de

\section{Evaluación del impacto clínico y la seguridad de una unidad de dolor torácico en pacientes con probabilidad baja e intermedia de síndrome coronario agudo}

\author{
Dagnovar Aristizábal O. ${ }^{\mathrm{a}, *}$, Cristina Montoya M. ${ }^{\mathrm{a}}$, Ángela M. Valencia C. ${ }^{\mathrm{a}}$, \\ Juan M. Jaime T. ${ }^{a}$ y Jaime A. Gallo V. ${ }^{a, b}$ \\ ${ }^{a}$ Centro Clínico y de Investigación SICOR, Soluciones Integrales en Riesgo Cardiovascular, Medellín, Colombia \\ ${ }^{\mathrm{b}}$ Facultad de Medicina, Universidad de Antioquia, Medellín, Colombia
}

Recibido el 29 de agosto de 2014; aceptado el 20 de mayo de 2015

Disponible en Internet el 30 de junio de 2015

\section{PALABRAS CLAVE \\ Dolor torácico; \\ Síndrome coronario \\ agudo; \\ Factores de riesgo; \\ Electrocardiograma; \\ Gestión sanitaria}

\begin{abstract}
Resumen
Antecedentes: Aunque las unidades de dolor torácico (UDT) permiten la estratificación y atención apropiada de los pacientes con dolor de pecho, en Colombia el desarrollo e implementación sistemática y estandarizada de este proceso de atención es escaso.

Objetivo: Evaluar el impacto clínico y la seguridad del proceso de atención de una UDT en pacientes con probabilidad baja e intermedia de síndrome coronario agudo en Medellín, Colombia.

Métodos: Estudio descriptivo que incluyó 277 pacientes, que fueron ingresados en una UDT de quienes se obtuvo información demográfica, clínica y de la gestión del proceso de atención. En el seguimiento a 30 días se evaluó la sobrevida y eventos compatibles con la enfermedad coronaria.

Resultados: De toda la muestra, el $13,0 \%$ de los pacientes fue remitido a un tercer nivel de atención y solo el $2,5 \%$ tuvieron un diagnóstico final confirmado de síndrome coronario agudo. La mediana del tiempo entre la recepción en urgencias y la toma del electrocardiograma fue 10,0 minutos, en el servicio de urgencias se calculó el puntaje TIMI y se hizo la estratificación del riesgo en el 85,5 y 73,9\%, respectivamente. La comparación entre la fase de implementación y consolidación mostró una mejoría en los indicadores $(\mathrm{p}<0,05)$. Durante el seguimiento a 30 días no se observaron muertes ni eventos coronarios.

Conclusión: La UDT basada en la evaluación clínica, la aplicación de la escala TIMI y la interpretación del electrocardiograma, constituyen una forma eficaz de estratificación de los pacientes con probabilidad baja o intermedia de síndrome coronario agudo, su implementación es segura y el riesgo de complicaciones es muy bajo.

(c) 2015 Sociedad Colombiana de Cardiología y Cirugía Cardiovascular. Publicado por Elsevier España, S.L.U. Este es un artículo Open Access bajo la licencia CC BY-NC-ND (http://creativecommons.org/licenses/by-nc-nd/4.0/).
\end{abstract}

* Autor para correspondencia.

Correo electrónico: dagnovar@une.net.co (D. Aristizábal O.). 


\section{KEYWORDS}

Chest pain;

Acute coronary

syndrome;

Risk factors;

Electrocardiogram;

Health management
Assessment of clinical impact and security of a chest pain unit in patients with low and intermediate probability of an acute coronary syndrome

\begin{abstract}
Background: Chest pain units (CPU) are an accepted method to assess patients admitted to an emergency department with chest pain with a potential ischemic origin. Yet in Colombia, the customary admission to a CPU in a standardized fashion is scarce and, to our knowledge, the outcome of implementing recognized protocols for chest pain has not been evaluated.

Objective: To assess the clinical impact and security of CPU, in patients with, low and intermediate probability of acute coronary syndrome, in Medellín, Colombia.

Methods: A descriptive study comprising 277 subjects who were consecutively admitted to the CPU. Variables included demographics, and performance measures to assess the process of care (timely action and process completion). A 30 days follow-up included survival and new admissions compatible with coronary syndromes.

Results: From the whole sample, $13.0 \%$ of patients were referred for hospitalization. However, only $2.5 \%$ had a final diagnosis of an acute coronary syndrome. Median time between reception and an electrocardiogram acquisition was 10 minutes TIMI score and acute coronary syndrome probability were reported in 85.5 and $73.9 \%$ of subjects respectively. Comparison between early implementation and consolidation phases showed a sensible improvement of performance measure indicators $(p<0.05)$. In addition, 30-day follow-up showed neither fatalities nor new coronary events.

Conclusion: Chest pain observation unit based on clinical assessment of thoracic pain, TIMI risk score and ST segment changes interpretation in the ECG is a safe and efficacious way to stratify low and intermediate chest pain leading to a safe patient discharge with very low risk of cardiovascular complications.

(C) 2015 Sociedad Colombiana de Cardiología y Cirugía Cardiovascular. Published by Elsevier España, S.L.U. This is an open access article under the CC BY-NC-ND license (http://creativecommons.org/licenses/by-nc-nd/4.0/).
\end{abstract}

\section{Introducción}

La unidad de dolor torácico (UDT) es un método innovador para la estratificación de pacientes con dolor en el tórax y se ha establecido como una alternativa a la hospitalización en pacientes con síntomas sugestivos de síndrome coronario agudo (SCA), sin evidencia objetiva de isquemia. La UDT permite asegurar una atención apropiada cuando se sospecha de dolor anginoso con un riesgo intermedio o bajo de $\mathrm{SCA}^{1,2}$. Aunque generalmente se describe como una unidad, la UDT debe ser considerada como un proceso de atención y no necesariamente como una entidad aparte dentro de un servicio de urgencias ${ }^{3}$. El cuidado se fundamenta en protocolos el cual puede ser proporcionado por médicos generales de urgencias entrenados, y su implementación exitosa requiere la coordinación cercana con un servicio de cardiología, al igual que se cuente con el apoyo del personal de otras áreas (enfermería, laboratorio clínico, radiología) ${ }^{4}$.

Existen diversos protocolos para realizar la estratificación e identificar los pacientes con dolor de pecho con un mayor riesgo de un $\mathrm{SCA}^{5-9}$. Estos se basan en las características clínicas y comportamiento hemodinámico de los pacientes, los hallazgos electrocardiográficos iniciales y el seguimiento del segmento ST y el perfil enzimático durante la estadía en la UDT. Con estas características se pueden tomar decisiones racionales acerca del nivel del cuidado médico requerido y el tipo de servicio de salud donde este debe realizarse ${ }^{10}$.

Como parte de la estratificación de la probabilidad de un SCA se ha utilizado el puntaje TIMI (por sus siglas en inglés, Thrombolysis In Myocardial Infarction) que se basa en la medición de siete variables cada una de las cuales suma un punto en el modelo matemático ${ }^{11}$. Aunque el puntaje fue desarrollado para la evaluación del riesgo en pacientes con SCA establecido, igualmente ha demostrado su valor pronóstico con relación a la mortalidad y desenlaces adversos mayores, en pacientes en el servicio de urgencias con sospecha de SCA ${ }^{12,13}$. Un puntaje TIMI alto (entre cinco a siete puntos) se asocia con una alta incidencia de muerte, infarto o necesidad de revascularización. No obstante, un puntaje bajo (entre cero a dos puntos) todavía tiene una frecuencia importante de eventos la cual es cercana al $2 \%$ en los siguientes 30 días $^{14}$. Por tanto, los pacientes con un puntaje TIMI bajo requieren de estrategias de estratificación adicionales ${ }^{15,16}$. Como se demostró recientemente, la combinación de un puntaje TIMI bajo, un electrocardiograma (ECG) normal y enzimas cardiacas no elevadas, tiene un valor predictivo negativo del $99,1 \%$, e identifica individuos con baja probabilidad de SCA que pueden ingresar en una UDT para observación o iniciar un seguimiento de forma ambulatoria ${ }^{9}$. En este modelo de atención se combinan el puntaje TIMI, la historia clínica, el examen físico, el ECG y los biomarcadores, para estratificar adecuadamente los pacientes que consultan al servicio de urgencias con dolor torácico y definir de forma apropiada el tratamiento.

Aunque en países desarrollados dicha metodología ha demostrado una alta capacidad de discriminación de los pacientes de bajo riesgo que pueden ser observados en la UDT y posteriormente ser dados de alta para seguimiento 
ambulatorio, de aquellos de alto riesgo que requieren una intervención inmediata ${ }^{4,17}$, apenas hace poco tiempo en Colombia, se ha iniciado su proceso de implementación. Sprockel et al. presentaron las rutas críticas para la evaluación y tratamiento del SCA en Bogotá ${ }^{18}$, pero actualmente no se conocen los resultados de la evaluación de estos procesos de atención.

Por tal razón, el objetivo de esta investigación fue evaluar el impacto clínico y la seguridad del proceso de atención de una UDT en pacientes con probabilidad baja e intermedia de SCA en la ciudad de Medellín, Colombia. Este trabajo presenta la implementación y desarrollo del protocolo de atención del dolor torácico, que sirvió de marco de referencia para la valoración, el diagnóstico y el tratamiento inicial de los pacientes en el servicio de urgencias de la IPS SURA Industriales. El protocolo reúne la evidencia existente con relación a las mejores prácticas actuales para asegurar que los pacientes reciban la atención adecuada, en el momento preciso, por el equipo adecuado. Se presentan los resultados de la evaluación del proceso después de 18 meses de implementación.

\section{Métodos}

Se realizó un estudio observacional, prospectivo y descriptivo, que incluyó 277 pacientes consecutivos con probabilidad baja e intermedia de SCA que fueron ingresados en la UDT del servicio de urgencias de la IPS SURA Industriales y coordinado por el Centro Clínico y de Investigación SICOR en Medellín, Colombia, durante los años 2011-2013. De los pacientes incluidos en el estudio se obtuvo información demográfica, clínica y de la gestión del proceso de atención en la UDT a partir de la historia clínica electrónica de la IPS SURA IPSA. Se hizo un seguimiento por historia clínica y telefónico del estado de los pacientes a los 30 días del alta de la UDT.

\section{Sujetos}

Pacientes que ingresaron en el servicio de urgencias con dolor torácico reciente o actual los cuales fueron evaluados y categorizados con una probabilidad baja o intermedia de SCA (fig. 1). Se incluyeron pacientes con malestar precordial resuelto (últimas 48 horas), angina de reciente inicio clase III de la Canadian Cardiovascular Society ${ }^{19}$, dolor atípico en personas con una edad igual o mayor a 65 años o diabéticos, con ECG normal y troponina inicial negativa, con un puntaje TIMI igual a cero, con enfermedad coronaria conocida, historia antigua mayor a tres meses de infarto de miocardio o revascularización con un episodio de dolor del tórax no sugestivo de enfermedad coronaria, con empeoramiento de la clase funcional y que no tenían una probabilidad alta de SCA después de la estratificación.

Se excluyeron pacientes con disnea, falla cardiaca, síncope en paciente menor de 30 años, dolor continuo o de localización dorsal, presencia de otra etiología señalada (disección aórtica, neumonía, pericarditis), los rayos $X$ de tórax anormales, la troponina basal elevada, las anormalidades electrocardiográficas concluyentes (cualquier suprao infradesnivel mayor o igual a un $\mathrm{mm}$ en dos derivaciones contiguas de la misma cara, compatibles con SCA) y las arritmias que requirieron tratamiento.

\section{Proceso de atención en la unidad de dolor torácico}

Los pacientes que ingresaron en el servicio de urgencias con dolor torácico siguieron el proceso de atención según una guía clínica estandarizada que apoyó el diagnóstico oportuno y la provisión de tratamiento teniendo en cuenta las mejores prácticas en quienes se sospecha un SCA (fig. 2).

Se emplearon herramientas tecnológicas que permitieron una comunicación cercana entre los médicos generales del servicio de urgencias y el cardiólogo del centro coordinador. El apoyo clínico por parte del cardiólogo fue realizado todos los días entre las 7 de la mañana y las seis de la tarde, mediante una línea telefónica y el acceso inmediato por Internet, con el fin de revisar la historia clínica electrónica y apoyar la toma de decisiones basadas en el protocolo de la guía clínica proporcionada. El paciente fue ubicado en un área con capacidad de monitorización continua (hemodinámica y del segmento ST) y la aplicación del tratamiento inicial según la estratificación del riesgo. En cada caso, se hizo intercambio de información entre el médico de urgencias y el cardiólogo del centro coordinador, sobre la historia clínica, los hallazgos en el examen físico, en el ECG y los resultados de laboratorio vía electrónica, sin requerir la atención presencial del cardiólogo en el servicio de urgencias.

La estratificación del riesgo de un SCA se realizó a partir de las características del dolor torácico, el puntaje TIMI y según las guías de la American Heart Association and American College of Cardiology, la cuales tienen en cuenta los signos y síntomas, los hallazgos en el ECG y los resultados de los biomarcadores ${ }^{4}$ (fig. 1).

Para la estratificación del riesgo de SCA se diseñó un formato con el fin de facilitar el proceso de registro en el servicio de urgencias que incluyó una definición de angina, la descripción de la presentación clínica, las características electrocardiográficas en tres categorías (la normal, los cambios mínimos y los cambios concluyentes) y los siete criterios del puntaje TIMI (fig. 1).

Después de la estratificación del riesgo de un SCA, aquellos pacientes estables hemodinámicamente y sin dolor precordial, sin evidencia objetiva de isquemia con un ECG normal o con cambios mínimos, y con un resultado negativo en el biomarcador (Roche Elecsys E170 analyser, F. Hoffmann-La Roche Ltd.) se consideraron de riesgo bajo o intermedio e ingresaron en la UDT para su seguimiento con un protocolo rápido que incluía por lo menos seis horas de observación y la realización de ECG y una segunda evaluación del biomarcador miocárdico.

A los pacientes que ingresaron en la UDT se les realizó una monitorización continua del ECG y la presión arterial, además, se les canalizó una vena periférica y se les administró oxígeno suplementario según saturación de oxígeno, $300 \mathrm{mg}$ de ácido acetilsalicílico, analgésicos y nitratos sublinguales si había dolor. También se les tomó una muestra de sangre para realizar el hemoleucograma y medir los niveles de creatinina y glucosa, además, se les realizó una radiografía simple de tórax posteroanterior y lateral. Durante la estancia en la UDT, se hizo un nuevo registro electrocardiográfico 


\begin{tabular}{|c|c|c|c|c|}
\hline \multicolumn{3}{|l|}{ Presentación clínica } & \multicolumn{2}{|c|}{ Características electrocardiográficas } \\
\hline \multicolumn{3}{|c|}{$\begin{array}{l}\square \text { Dolor anginoso* prolongado (> 15-20 min) y en reposo } \\
\text { (alta probabilidad de IAM) } \\
\square \text { Angina nueva, clasificación II y III Canadiense, o } \\
\text { desestabilización reciente de angina previamente } \\
\text { estable clase III Canadiense } \\
\square \text { No hay dolor actual pero con angina previa típica: } \\
\text { - Molestia opresiva en tórax, mandíbula, brazos. } \\
\text { - Precipitada por ejercicio físico o estrés sicológico } \\
\text { - Mejora con reposo o nitratos en unos } 5 \text { minutos }\end{array}$} & \multicolumn{2}{|c|}{$\begin{array}{l}\square \text { Normal (40\%) } \\
\square \text { Cambios mínimos: cambios del ST ( } \leq 0,5 \mathrm{~mm}) \text {, trastorno } \\
\text { de la conducción intraventricular, hipertrofia } \\
\text { ventricular izquierda, cubeta digitálica. } \\
\square \text { Cambios concluyentes (alta probabilidad): } \\
\text { - Infradesnivel del segmento ST } \geq 1 \mathrm{~mm} \text { en dos } \\
\text { derivaciones contiguas ( } 20 \%) \text {. } \\
\text { - Inversión de la onda T, marcada ( } \geq 2 \mathrm{~mm} \text { ) y } \\
\text { asimétrica (25\%). }\end{array}$} \\
\hline \multicolumn{5}{|l|}{ Puntaje TIMI } \\
\hline \multicolumn{3}{|c|}{$\begin{array}{l}\square \text { Edad } \geq 65 \text { años } \\
\square \geq 3 \text { factores de riesgo (historia familiar, HTA, } \\
\text { hiperlipidemia, diabetes mellitus, tabaquismo } \\
\square \text { Uso de ASA en los siete días previos } \\
\square \text { Enfermedad coronaria conocida (estenosis coronaria } \\
>50 \% \text { no tratada, revascularización previa) } \\
\square>1 \text { episodio de angina en las últimas } 24 \text { horas } \\
\square \text { Desviación del segmento } S T \geq 0,5 \mathrm{~mm} \\
\square \text { Elevación de biomarcadores (troponina) }\end{array}$} & \multicolumn{2}{|c|}{$\begin{array}{l}\text { Dolor anginoso es molestia u opresión retroesternal } \\
\text { difusa, con o sin irradiación, severo o no, con sudoración } \\
\text { y/o náuseas-vómito, que no lo afectan los cambios de } \\
\text { posición, movimiento o respiración. Puede ser atípico (en } \\
\text { epigastrio, en cuello o brazos) en }<40 \text { y > } 70 \text { años, en } \\
\text { mujeres, diabetes millitus o insuficiencia renal crónica. A } \\
\text { veces simula dispepsia o puede predominar la disnea. }\end{array}$} \\
\hline Característica & Alta probabilidad & $\square$ & Intermedia probabilidad & Baja probabilidad \\
\hline Historia clínica & \begin{tabular}{|c|} 
Molestia o dolor en el pecho o en el brazo \\
izquierdo como síntoma principal que \\
reproduce angina previamente \\
documentada. Historia conocida de \\
enfermedad coronaria, incluyendo infarto \\
de miocardio.
\end{tabular} & & $\begin{array}{l}\text { Molestia o dolor en el pecho o en } \\
\text { el brazo izquierdo como síntoma } \\
\text { principal. } \\
\text { Edad } \geq 65 \text { años } \\
\text { Hombre } \\
\text { Diabetes mellitus } \\
\end{array}$ & $\begin{array}{l}\text { Síntomas isquémicos probables en } \\
\text { ausencia de las características } \\
\text { intermedias. } \\
\text { Uso reciente de cocaína }\end{array}$ \\
\hline Examen físico & $\begin{array}{c}\text { Soplo de insuficiencia mitral transitorio, } \\
\text { hipotensión, diaforesis, edema pulmonar } \\
\text { o crépitos. }\end{array}$ & & $\begin{array}{l}\text { Paciente estable y sin dolor } \\
\text { precordial. } \\
\text { fermedad vascular extracardíaca }\end{array}$ & $\begin{array}{l}\text { Molestia precordial reproducible con } \\
\text { la palpación }\end{array}$ \\
\hline Electrocardiografía & $\begin{array}{c}\text { Desviación transitoria nueva o } \\
\text { posiblemente nueva del ST }(\geq 0,5 \mathrm{~mm}) \\
\text { o inversión de la onda T en múltiples } \\
\text { derivadas. }\end{array}$ & & $\begin{array}{l}\text { Ondas Q fijas. } \\
\text { presión del } \mathrm{ST} \text { de } 0,5 \mathrm{~mm} \text { a } 1 \mathrm{~mm} \text {. } \\
\text { Inversión de la onda } \mathrm{T}>1 \mathrm{~mm} \text {. }\end{array}$ & $\begin{array}{c}\text { Aplanamiento de la onda } T \text { o inversión } \\
<1 \mathrm{~mm} \text { en derivaciones con ondas } \mathrm{R} \\
\text { dominantes o ECG normal. }\end{array}$ \\
\hline Biomarcadores & Troponina I o CPK-MB elevada & & Normal & Normal \\
\hline
\end{tabular}

Figura 1 Formato para la estratificación del riesgo de un síndrome coronario agudo en el servicio de urgencias según la historia clínica, el examen físico, los hallazgos en el electrocardiograma, los resultados de los biomarcadores y el puntaje TIMI. ASA: ácido acetilsalicílico; CPK-MB: creatín-fosfoquinasa fracción MB; ECG: electrocardiograma; HTA: hipertensión arterial; IAM: infarto agudo del miocardio; TIMI: Thrombolysis In Myocardial Infarction.

Adaptado de: Amsterdam $\mathrm{EA}^{4}$.

y medición de las troponinas a las seis horas del ingreso. Según la evolución, en caso de aparición de nuevos síntomas o dolor, se realizaron evaluaciones más frecuentes, cada dos horas, con ECG y nueva reestratificación.

En aquellos pacientes cuyos estudios fueron negativos durante las seis horas de seguimiento en la UDT, se les dio de alta con remisión para estratificación no invasiva del riesgo de insuficiencia coronaria por medio de pruebas diagnósticas ambulatorias (prueba de esfuerzo electrocardiográfica, ecocardiografía de estrés o prueba de perfusión miocárdica) en las próximas 48-72 horas, además, según criterio clínico se inició terapia de antiagregación plaquetaria con ácido acetilsalicílico $100 \mathrm{mg}$ /día, betabloqueador o nitrato oral hasta la cita de evaluación por cardiología. En los pacientes en los que fue preciso descartar otras etiologías cardiacas de dolor torácico no coronario y valorar la contractilidad segmentaria y la función ventricular izquierda, se les realizó una ecocardiografía Doppler. La realización de estos procedimientos fue ambulatoria y la asignación de las citas se hizo al momento del alta del servicio de urgencias. En caso de no poder realizarse las pruebas diagnósticas o en presencia de otra sospecha diagnóstica, el paciente se hospitalizó.

Aquellos pacientes quienes durante el seguimiento en la UDT desarrollaron características de alto riesgo, cambios en el ECG, en los biomarcadores, presentaron pruebas confirmatorias alteradas, inestabilidad hemodinámica, requirieron de nitratos por vía intravenosa y soporte 


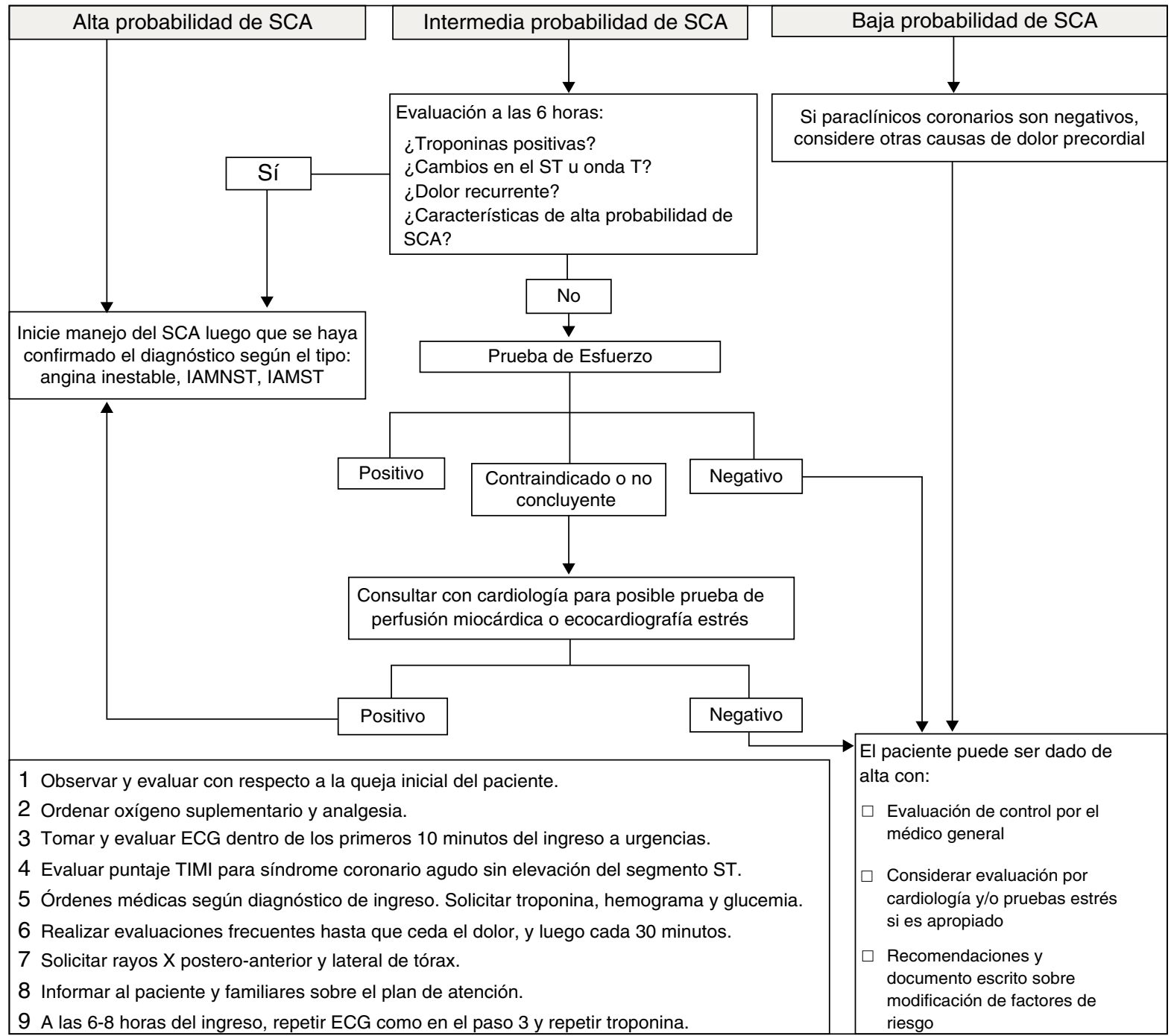

Figura 2 Algoritmo de la guía clínica estandarizada, según la estratificación del riesgo de síndrome coronario agudo, de los pacientes que ingresaron en el servicio de urgencias con dolor torácico. ECG: electrocardiograma; IAMNST: infarto agudo del miocardio sin elevación del segmento ST; IAMST: infarto agudo del miocardio con elevación del segmento ST; SCA; síndrome coronario agudo; TIMI: Thrombolysis In Myocardial Infarction.

hemodinámico, o se les encontró una auscultación cardiaca o pulmonar anormal, también fueron remitidos para ser hospitalizados por la alta probabilidad de SCA.

\section{Seguimiento ambulatorio}

Una vez completado el protocolo, que incluyó la cita por cardiología posterior a la estadía en la UDT, se realizó el seguimiento mediante la historia clínica electrónica de la utilización de los servicios de consulta externa, urgencias y hospitalización durante los siguientes 30 días. Adicionalmente, se verificó mediante llamada telefónica el estado del individuo (vivo o muerto) y si ocurrieron episodios compatibles con la enfermedad coronaria, en el mismo período de seguimiento, como una nueva hospitalización por dolor de pecho, infarto de miocardio y la necesidad de un procedimiento de intervencionismo cardiaco.

\section{Indicadores de oportunidad de la atención, la actuación y el resultado de la unidad de dolor torácico}

Como medidas del impacto clínico y de seguridad del proceso de atención en la UDT se usaron indicadores de la oportunidad, la actuación y el resultado.

Los indicadores de oportunidad en la atención fueron definidos a partir de los tiempos de acción en minutos desde el ingreso del paciente al servicio de urgencias.

Como indicadores de la actuación se definieron el porcentaje de pacientes que recibieron $300 \mathrm{mg}$ de ácido acetilsalicílico, en quienes se les calculó el puntaje TIMI, se reportó la estratificación de riesgo de SCA, se les tomó la radiografía de tórax, se les midió los niveles de la troponina al ingreso y a las seis horas y aquellos que tenían una nota de seguimiento en la historia clínica a las dos y seis 
horas del ingreso en la UDT. También se calculó el porcentaje de pacientes en cada nivel de estratificación, la concordancia de las lecturas del ECG entre el médico del servicio de urgencias y el cardiólogo del centro coordinador, el porcentaje de pacientes con una prueba diagnóstica ambulatoria postegreso de la UDT y el porcentaje de pacientes con un diagnóstico final confirmado de SCA.

Como indicadores de resultado se usaron el porcentaje de pacientes vivos a los 30 días del ingreso en la UDT y el porcentaje de pacientes quienes presentaron nuevos episodios compatibles con la enfermedad coronaria.

\section{Aspectos éticos}

Este estudio fue clasificado como una investigación de riesgo mínimo, por tratarse de la aplicación de un protocolo científicamente estandarizado, que incluyó la revisión de historias clínicas, el cual fue aprobado por los comités de las instituciones participantes. Se promulgó el respeto, la justicia y la beneficencia a las personas de acuerdo con las normas científicas, técnicas y administrativas para la investigación en salud del Ministerio de la Protección Social de Colombia en la Resolución 008430 del año $1993^{20}$, además, se tuvieron en cuenta los principios de la declaración de Helsinki en su última revisión ${ }^{21}$.

\section{Análisis estadístico}

Se utilizó la prueba de Kolmogorov-Smirnov para evaluar si las variables provenían de una población con distribución normal. Para la descripción de las variables cuantitativas se utilizaron el promedio, la desviación estándar, la mediana y el rango intercuartílico. Igualmente, para la descripción de las variables nominales se utilizaron proporciones. Para comparar los indicadores de oportunidad en la atención, entre los pacientes que ingresaron en la UDT en la fase de implementación (primeros seis meses) y aquellos ingresados en la fase de consolidación (últimos doce meses), se utilizó la prueba $U$ de Mann Whitney. También se utilizó la prueba $\chi^{2}$ de independencia para comparar las frecuencias de los indicadores de actuación entre los pacientes de la fase de implementación y aquellos de la fase de consolidación. Para los análisis de concordancia interobservador de los hallazgos en el ECG entre el médico del servicio de urgencias y el cardiólogo del centro coordinador se utilizó el índice Kappa. Se definieron las categorías de normal, cambios mínimos y cambios concluyentes en el ECG. Para todos los análisis se utilizó un nivel de significación estadística $\alpha=0,05$ y se usó el software EPIDAT 4,0 de la Organización Panamericana de la Salud e IBM SPSS Statistics, versión 21.0.

\section{Resultados}

Se incluyeron 277 pacientes en la UDT, con una edad promedio de 47,4 $\pm 13,2$ años, de los cuales el 59,6\% $(n=165)$ eran de sexo masculino. Al ingreso la probabilidad de SCA fue baja en el 79,7\% e intermedia en el 20,3\% de los pacientes. Se reportó un puntaje de $\mathrm{TIMI}=0$ en el $67,5 \%, \mathrm{TIMI}=1$ en el $19,0 \%, \mathrm{TIMI}=2$ en el 7,6\%, TIMI $=3$ en el 5,5\% y $\mathrm{TIMI}=4$ en el $0,4 \%$. Entre los pacientes ingresados en la UDT, a quienes se les hizo el seguimiento durante seis horas, el diagnóstico final después del período de observación fue un SCA con ST elevado en el 1,8\% (n=5), un SCA sin ST elevado en el $2,9 \%(n=8)$, un SCA probable en el $73,3 \%(n=203)$ y un dolor torácico de origen no cardiaco en el $22,0 \%(n=61)$.

De toda la muestra, solo el $13,0 \%(n=36)$ de los pacientes fue remitido a un tercer nivel de atención; de los pacientes remitidos a hospitalización, un 19,4\% $(n=7)$ tuvieron evidencia de un SCA. La tabla 1 presenta los diagnósticos definitivos de los pacientes remitidos al tercer nivel de atención. En los demás pacientes incluidos en la UDT, que corresponde al $87,0 \%(n=241)$, se descartó un SCA y fueron dados de alta después de completar el protocolo (estancia de seis horas en la UDT, la realización de la prueba diagnóstica ambulatoria y la cita de evaluación por cardiología). El porcentaje de pacientes a quienes se les realizó una prueba diagnóstica ambulatoria fue del 45,4\% $(n=126)$.

Con relación a los indicadores de oportunidad en la atención la mediana del tiempo entre la recepción en urgencias y la priorización fue 7,6 minutos (rango, 2,8-13,3), del tiempo entre la recepción en urgencias y la toma del ECG fue 10,0 minutos (rango, 4,4-27,9) y del tiempo de llegada del ECG al centro coordinador y la respuesta fue 25,8 minutos (rango, 18,0-37,5). En la tabla 2 se describen los tiempos en minutos, de los demás indicadores de oportunidad, desde el ingreso del paciente al servicio de urgencias.

En los indicadores de la actuación, globalmente el porcentaje de pacientes en quienes se les calculó el puntaje TIMI fue del $85,5 \%(n=236)$, se reportó la estratificación de riesgo de SCA que fue del $73,9 \%(n=204)$, se les tomó la radiografía de tórax que fue del $76,5 \%(n=212)$, se les midió los niveles de la troponina al ingreso, fue del 97,1\% $(n=269)$ y a las seis horas fue del 61,4\% $(n=170)$, tenían el reporte en la historia clínica de la troponina al ingreso, fue del $88,4 \%$ $(n=245)$ y a las seis horas fue del $58,5 \%(n=162)$, recibieron $300 \mathrm{mg}$ de ácido acetilsalicílico fue del 52,0\% ( $n=143)$, y aquellos que tenían la nota de seguimiento en la historia clínica a las dos horas del ingreso a la UDT fue del $85,9 \%$ $(n=238)$ y a las seis horas del ingreso a la UDT fue del $69,3 \%$ $(\mathrm{n}=192)$ (tabla 3).

Cuando se realizó la comparación entre los pacientes que ingresaron en la UDT en una fase de implementación $(n=96)$ y aquellos que ingresaron en la UDT en la fase de consolidación $(n=181)$, se observó una reducción de la mediana del tiempo de llegada del ECG al centro coordinador y la respuesta de 33,6 minutos a 23,1 minutos $(p<0,001)$ (tabla 2) y un aumento en la proporción de pacientes a quienes se les calculó el puntaje TIMI de 65,3 a 96,1\% ( $p<0,001)$, se reportó la estratificación de riesgo de SCA de 43,2 a $90,1 \%$ $(\mathrm{p}<0,001)$, se les tomó la radiografía de tórax de 54,2 a $88,4 \%(p<0,001)$, se les midió los niveles de la troponina al ingreso de 93,8 a 98,9\% ( $p=0,015)$ y a las seis horas de 39,6 a $72,9 \%(p<0,001)$, tenían el reporte de la troponina en la historia clínica a las seis horas de 35,4 a 70,7\% ( $<<0,001)$ y aquellos que tenían la nota de seguimiento en la historia clínica a las dos horas del ingreso a la UDT de 75,0 a $91,7 \%$ $(\mathrm{p}<0,001)$ y a las seis horas del ingreso a la UDT de 52,9 a $82,6 \%(p<0,001)$ (tabla 3$)$.

En la evaluación de la concordancia interobservador, de los hallazgos en el ECG, entre el médico del servicio de urgencias y el cardiólogo del centro coordinador, se observó un acuerdo del 71,4\% y un índice Kappa de 0,46 (IC 95\% entre $0,33-0,58 ; p<0,001)$. 
Tabla 1 Comparación entre el diagnóstico definitivo en el hospital de tercer nivel y el diagnóstico al final de las seis horas de seguimiento en la unidad de dolor torácico de los pacientes remitidos

\begin{tabular}{|c|c|c|}
\hline $\begin{array}{l}\text { Diagnóstico definitivo en el hospital de tercer nivel } \\
(n=36)\end{array}$ & $\begin{array}{l}\text { Diagnóstico al final de las seis horas de } \\
\text { seguimiento en la UDT }(n)\end{array}$ & Porcentaje $(n)$ \\
\hline Infarto del miocardio & $\begin{array}{l}\text { SCA con elevación del ST (1) } \\
\text { SCA sin elevación del ST (2) } \\
\text { SCA probable (1) }\end{array}$ & $11,1(4)$ \\
\hline Miocardiopatía de Takotsubo & SCA sin elevación del ST (1) & $2,8(1)$ \\
\hline Angina de pecho & $\begin{array}{l}\text { SCA sin elevación del ST (1) } \\
\text { SCA probable (1) }\end{array}$ & $5,6(2)$ \\
\hline Dolor torácico no cardiaco & $\begin{array}{l}\text { SCA sin elevación del ST (1) } \\
\text { Dolor torácico de origen no cardiaco (1) }\end{array}$ & $5,6(2)$ \\
\hline Dolor torácico inespecífico & $\begin{array}{l}\text { SCA con elevación del ST (4) } \\
\text { SCA sin elevación del ST (3) } \\
\text { SCA probable (5) } \\
\text { Dolor torácico de origen no cardiaco (4) }\end{array}$ & $44,4(16)$ \\
\hline $\begin{array}{l}\text { Causa cardiaca no isquémica (arritmia, insuficiencia } \\
\text { cardiaca congestiva, valvulopatía) }\end{array}$ & SCA probable $(3)$ & $8,3(3)$ \\
\hline Dolor gastrointestinal & $\begin{array}{l}\text { SCA probable }(1) \\
\text { Dolor torácico de origen no cardiaco (2) }\end{array}$ & $8,3(3)$ \\
\hline Dolor músculo-esquelético & Dolor torácico de origen no cardiaco (1) & $2,8(1)$ \\
\hline Urgencia hipertensiva & Dolor torácico de origen no cardiaco (3) & $8,3(3)$ \\
\hline Accidente cerebrovascular & Dolor torácico de origen no cardiaco (1) & $2,8(1)$ \\
\hline
\end{tabular}

SCA: síndrome coronario agudo; UDT: unidad de dolor torácico.

De todos los pacientes incluidos en la UDT, no fue posible hacer un contacto telefónico posterior al alta en el 7,2\% $(n=20)$. En el seguimiento a 30 días, no se identificaron muertes ni eventos coronarios.

\section{Discusión}

El dolor torácico representa hasta una cuarta parte del volumen de atención en un servicio de urgencias. La mayoría de estos pacientes se presentan con etiologías no cardiacas. No obstante, aun entre los que parecen tener un bajo riesgo de un SCA, un 1 a $3 \%$ pueden presentar un infarto agudo de miocardio y ser erróneamente dados de alta ${ }^{13,22,23}$. Los errores diagnósticos en la identificación de esta población pueden generar eventos adversos graves o fatales ${ }^{22}$. Para aminorar la probabilidad de estos errores diagnósticos se han propuesto evaluaciones sistemáticas de los pacientes que consultan por dolor torácico o síntomas sugestivos de isquemia miocárdica. Estas evaluaciones en el servicio de urgencias se han realizado basadas en esquemas de estratificación de riesgo y en un marco de tiempo definido ${ }^{4,9,24,25}$. Con esta metodología se diseñó el presente estudio.

Los hallazgos principales muestran que es posible implementar en forma segura una UDT con el apoyo remoto del especialista en cardiología para la estratificación correcta de esta población. En la presente investigación solo el 13\% de los pacientes fueron remitidos a hospitalización, se tuvo un promedio de eventos del $2,5 \%$ y no se presentó ningún fallecimiento.

Desde hace más de tres décadas las UDT han demostrado que mejoran los desenlaces clínicos y son costoefectivas $^{26,27}$. En diversos países del mundo se han propuesto protocolos para el tipo de población que debe ingresar en estas unidades ${ }^{1,4,25,28}$. En el estudio ASPECT $^{9}$ utilizaron un protocolo rápido con un esquema de estratificación similar al nuestro y demostraron que las características clínicas, las electrocardiográficas, la aplicación del puntaje TIMI, más una estancia corta en urgencias, tuvo un valor predictivo negativo del $99,1 \%$ con una frecuencia de eventos adversos solo del $0,9 \%$. En nuestro estudio no se observó ningún evento adverso dentro los siguientes 30 días a la salida de la UDT, teniendo en cuenta que se admitieron a la unidad de observación pacientes con probabilidad baja e intermedia de un SCA. Además, es importante considerar, que la entrada a una UDT significa el completar el proceso de atención, el cual incluye descartar la presencia de isquemia en el ECG y una segunda medición de la troponina negativa a las seis horas ${ }^{29}$. En el presente protocolo al paciente se le dio de alta con una cita para una prueba de esfuerzo ambulatoria (con o sin imágenes según el ECG) y una consulta de cardiología prioritaria dentro de las siguientes 48 a 72 horas (fig. 2). La adherencia a este proceso de atención fue incompleta debido a que solo al $45,4 \%$ de los pacientes se les realizó la prueba diagnóstica ambulatoria. El estudio de Richards et al. ${ }^{30}$ reportó mayores promedios de cumplimiento de la prueba diagnóstica y de la cita de valoración por cardiología, cuando la prueba fue agendada previo al alta del paciente. Este no fue siempre posible en nuestra muestra de pacientes.

Previo al inicio de la UDT se requirió de la capacitación del grupo asistencial de urgencias para uniformizar la evaluación del dolor torácico y las capacidades de lectura del ECG, así como también, la estandarización de la aplicación de la guía clínica la cual debía incluir el reporte sistemático de la probabilidad de SCA en los pacientes. Este proceso 
Tabla 2 Descripción de los indicadores de oportunidad en la atención definidos a partir de los tiempos de acción en minutos desde el ingreso del paciente en el servicio de urgencias en toda la muestra y comparación entre los pacientes que ingresaron en la unidad de dolor torácico en la fase de implementación (primeros seis meses) y aquellos ingresados en la fase de consolidación (últimos doce meses)

\begin{tabular}{|c|c|c|c|c|c|c|c|c|c|c|}
\hline \multirow[t]{2}{*}{ Indicadores de oportunidad en la atención } & \multicolumn{3}{|c|}{ Implementación ( $n=96)$} & \multicolumn{3}{|c|}{ Consolidación $(n=181)$} & \multicolumn{3}{|c|}{ Total $(n=277)$} & \multirow[t]{2}{*}{ Valor $\mathrm{p}$} \\
\hline & Mediana & p 25 & p 75 & Mediana & p 25 & p 75 & Mediana & p 25 & p 75 & \\
\hline Recepción en urgencias-priorización (min) & 8,1 & 3,5 & 13,4 & 7,4 & 2,5 & 13,3 & 7,6 & 2,8 & 13,3 & 0,500 \\
\hline $\begin{array}{l}\text { Priorización-atención del médico de urgencias } \\
(\mathrm{min})\end{array}$ & 12,0 & 6,8 & 19,8 & 13,3 & 7,0 & 21,3 & 13,1 & 7 & 20,6 & 0,179 \\
\hline $\begin{array}{l}\text { Recepción en urgencias-atención del médico de } \\
\text { urgencias (min) }\end{array}$ & 20,9 & 13,1 & 31,1 & 20,5 & 14,7 & 35,1 & 20,5 & 13,5 & 33,5 & 0,304 \\
\hline $\begin{array}{l}\text { Recepción en urgencias-toma del } \\
\text { electrocardiograma (min) }\end{array}$ & 9,4 & 4,4 & 22,2 & 11,3 & 4,3 & 30,0 & 10,0 & 4,4 & 27,9 & 0,482 \\
\hline $\begin{array}{l}\text { Llegada del electrocardiograma al centro } \\
\text { coordinador-respuesta del electrocardiograma } \\
\text { del centro coordinador (min) }\end{array}$ & 33,6 & 21,8 & 53,4 & 23,1 & 15,4 & 32,8 & 25,8 & 18 & 37,5 & $<0,001$ \\
\hline $\begin{array}{l}\text { Recepción en urgencias-toma de la muestra de } \\
\text { sangre para troponinas }(\mathrm{min})\end{array}$ & 54,8 & 41,4 & 75,0 & 58,4 & 43,5 & 77,0 & 57,0 & 42,1 & 76,5 & 0,477 \\
\hline $\begin{array}{l}\text { Toma de la muestra de sangre para } \\
\text { troponinas-reporte del resultado en la historia } \\
\text { clínica (min) }\end{array}$ & 152,5 & 116,2 & 181,5 & 155,9 & 121,3 & 203,5 & 153,7 & 118 & 192,2 & 0,148 \\
\hline
\end{tabular}

\footnotetext{
" Prueba U de Mann Whitney, comparación entre los pacientes que ingresaron en la unidad de dolor torácico en la fase de implementación (primeros seis meses) y aquellos ingresados
} en la fase de consolidación (últimos doce meses). 
Tabla 3 Descripción de los indicadores de actuación en toda la muestra y comparación entre los pacientes que ingresaron en la unidad de dolor torácico en la fase de implementación (primeros seis meses) y aquellos ingresados en la fase de consolidación (últimos doce meses)

\begin{tabular}{|c|c|c|c|c|}
\hline Indicadores de actuación & $\begin{array}{l}\text { Implementación }(n=96) \\
\%\end{array}$ & $\begin{array}{l}\text { Consolidación }(n=181) \\
\%\end{array}$ & $\begin{array}{l}\text { Total }(n=277) \\
\%\end{array}$ & Valor $\mathrm{p}^{*}$ \\
\hline Se calculó el puntaje TIMI & 65,3 & 96,1 & 85,5 & $<0,001$ \\
\hline Se hizo la estratificación de riesgo de SCA & 43,2 & 90,1 & 73,9 & $<0,001$ \\
\hline Se tomó la radiografía de tórax & 54,2 & 88,4 & 76,5 & $<0,001$ \\
\hline Se tomó la troponina al ingreso & 93,8 & 98,9 & 97,1 & 0,015 \\
\hline Se tomó la troponina a las seis horas & 39,6 & 72,9 & 61,4 & $<0,001$ \\
\hline $\begin{array}{l}\text { Se reportó en la historia clínica la troponina } \\
\text { al ingreso }\end{array}$ & 87,5 & 89,0 & 88,4 & 0,719 \\
\hline $\begin{array}{l}\text { Se reportó en la historia clínica la troponina } \\
\text { a las seis horas }\end{array}$ & 35,4 & 70,7 & 58,5 & $<0,001$ \\
\hline Se formuló 300 mg de ácido acetilsaliscílico & 46,8 & 54,7 & 52,0 & 0,214 \\
\hline $\begin{array}{l}\text { Se hizo la nota de seguimiento en la historia } \\
\text { clínica a las dos horas }\end{array}$ & 75,0 & 91,7 & 85,9 & $<0,001$ \\
\hline $\begin{array}{l}\text { Se hizo la nota de seguimiento en la historia } \\
\text { clínica a las seis horas }\end{array}$ & 52,9 & 82,6 & 73,0 & $<0,001$ \\
\hline
\end{tabular}

SCA: síndrome coronario agudo; TIMI: Thrombolysis In Myocardial Infarction.

" Prueba $\chi^{2}$ de independencia, comparación entre los pacientes que ingresaron en la unidad de dolor torácico en la fase de implementación (primeros seis meses) y aquellos ingresados en la fase de consolidación (últimos doce meses).

ha demostrado que mejora los desenlaces de los pacientes en el servicio de urgencias $^{31}$. Como pudo verse en nuestro estudio este protocolo puede tardar hasta seis meses en implementarse. La ayuda de plataformas tecnológicas dedicadas para el reporte de esta información puede acelerar su implementación.

La ejecución del proceso de atención se logró con el apoyo remoto del especialista en cardiología, con el uso de tecnologías de información, para asegurar el estándar de cuidado clínico y validar la interpretación del ECG por el médico general en urgencias y la estratificación realizada, con el fin de soportar la toma de decisiones fundamentadas en la guía clínica ${ }^{4}$. Algunos de los indicadores de desempeño de este proceso fueron objeto de optimización en la fase de consolidación. Se observó que algunos de los tiempos de acción cambiaron desde la fase de implementación a la fase de consolidación, en particular fue posible mejorar los tiempos de respuesta de la lectura del ECG por el especialista y el reporte sistemático del puntaje TIMI, la estratificación de riesgo de $\mathrm{SCA}$, la toma de la radiografía de tórax y la medición de las troponinas. En la actualidad se evalúa cómo la historia clínica alojada en la «web» o la «nube» puede mejorar la lectura asincrónica de los ECG para llevarlas a tiempo real.

Como lo propone Mendoza, después de estructurar un estándar de cuidado del dolor torácico en urgencias, es necesario lograr la implementación, el seguimiento, la evaluación de la adherencia y la medición de un antes y un después, para determinar el impacto real de estas herramientas en una institución ${ }^{32}$. Según nuestro conocimiento, esta investigación presenta por primera vez los resultados de la evaluación de una UDT en el país. Igualmente demuestran que el presente protocolo es seguro y puede ser rápidamente implementado en servicios de urgencias generales con el apoyo remoto de un especialista en cardiología.
Se han evaluado otras estrategias para mejorar el triaje del paciente con dolor torácico en el servicio de urgencias; en particular se ha considerado si se requiere de la utilización de imágenes cardiacas como la angiografía coronaria con la tomografía para la toma de decisiones. Recientemente, varios autores evaluaron la utilización de este procedimiento comparado con el cuidado estándar sin imágenes como se hizo en nuestro protocolo ${ }^{33-35}$. Los hallazgos indican que si bien este procedimiento puede acortar el tiempo de estancia en el servicio de urgencias, aumenta la utilización de otras ayudas diagnósticas y los costos de atención sin mejorar los desenlaces clínicos ${ }^{34}$. Al momento, el método utilizado en nuestro estudio basado en una buena evaluación clínica del dolor torácico, más la aplicación de la escala TIMI y la interpretación acertada de los cambios en el segmento ST en el ECG, constituyen la mejor forma de estratificación de los pacientes con probabilidad baja 0 intermedia de $\mathrm{SCA}^{36}$; los presentes resultados corroboran su seguridad y buen desempeño.

Nuestro estudio tiene algunas limitaciones debido a que es un reporte de un solo centro (una IPS general que atiende personas con aseguramiento privado) y puede no reflejar la población afiliada al Sistema General de Seguridad Social. Además, la muestra incluida en general fue joven con una baja probabilidad pretest de enfermedad coronaria lo cual se reflejó en una baja frecuencia de SCA en los pacientes que fueron remitidos a hospitalización. Además, no fue posible hacer un seguimiento a 30 días a todas las personas; sin embargo, unas pérdidas menores al $10 \%$ están dentro de los esperado en este tipo de estudios.

La prueba diagnóstica ambulatoria, para descartar de forma definitiva la ausencia de insuficiencia coronaria, no fue realizada en todos los pacientes después de las seis horas de observación en la UDT. A pesar de esto, no se presentaron muertes ni eventos coronarios, posiblemente relacionados 
con las características demográficas de la muestra de estudio. Como el cálculo de los tiempos de acción se hizo a partir de los registros de historia clínica electrónica, algunos de los indicadores estuvieron sujetos a la consignación de esta información. Este hecho fue particularmente notorio para el reporte de la troponina lo cual afectó este tiempo trazador. No obstante, la toma de decisiones en el servicio de urgencias se realizó con base en el informe de este biomarcador directamente desde el laboratorio clínico.

\section{Conclusiones}

La UDT es un proceso de atención eficaz para el triaje del dolor precordial, su implementación basada en la evaluación clínica del dolor torácico, la aplicación de la escala TIMI y la interpretación acertada de los cambios del segmentos ST en el ECG es segura y el riesgo de complicaciones es muy bajo cuando la probabilidad de SCA es baja o intermedia. El uso de las tecnologías de información para el apoyo remoto de la cardiología, que incluya la misma historia clínica electrónica puede optimizar los indicadores de gestión y los tiempos trazadores del proceso de cuidado. Un factor crítico de éxito es la interiorización de la guía clínica estandarizada por el grupo asistencial de urgencias encargado del cuidado de los pacientes.

\section{Responsabilidades éticas}

Protección de personas y animales. Los autores declaran que para esta investigación no se han realizado experimentos en seres humanos ni en animales.

Confidencialidad de los datos. Los autores declaran que han seguido los protocolos de su centro de trabajo sobre la publicación de datos de pacientes.

Derecho a la privacidad y consentimiento informado. Los autores declaran que en este artículo no aparecen datos de pacientes.

\section{Conflicto de intereses}

Los autores declaran no tener ningún conflicto de intereses.

\section{Financiación}

Esta investigación fue realizada con recursos de la IPS SURA Industriales y el Centro Clínico y de Investigación SICOR, Soluciones Integrales en Riesgo Cardiovascular, Medellín (Colombia).

\section{Agradecimientos}

Los autores agradecen al personal médico, de enfermería y administrativo de la IPS SURA Industriales y la Gerencia de Salud de Suramericana por su dedicación y excelente acompañamiento para la realización de la presente investigación.

También agradecen a los médicos generales Jon Kepa Balparada, María Fernanda Corrales, Lina María Morera,
Alejandra Medina, Silvana Chimá y Fair Clavijo; los cardiólogos Carlos Cubides, Jorge Ferreira, Salim Ahumada y Juan Carlos Aristizábal; así como al grupo de enfermería de SICOR, el esmerado trabajo asistencial durante la ejecución del proyecto.

\section{Bibliografía}

1. Farkouh ME, Smars PA, Reeder GS, Zinsmeister AR, Evans RW, Meloy TD, et al. A clinical trial of a chest-pain observation unit for patients with unstable angina. Chest Pain Evaluation in the Emergency Room (CHEER) Investigators. N Engl J Med. 1998;339(26):1882-8.

2. Clancy M. Chest pain units. BMJ. 2002;325(7356):116-7.

3. Hutter AM Jr, Amsterdam EA, Jaffe AS. 31st Bethesda Conference. Emergency Cardiac Care. Task force 2: Acute coronary syndromes: Section 2B-Chest discomfort evaluation in the hospital. J Am Coll Cardiol. 2000;35(4):853-62.

4. Amsterdam EA, Kirk JD, Bluemke DA, Diercks D, Farkouh ME, Garvey JL, et al. Testing of low-risk patients presenting to the emergency department with chest pain: a scientific statement from the American Heart Association. Circulation. 2010;122(17):1756-76.

5. Goldman L, Cook EF, Brand DA, Lee TH, Rouan GW, Weisberg MC, et al. A computer protocol to predict myocardial infarction in emergency department patients with chest pain. N Engl J Med. 1988;318(13):797-803.

6. Goldman L, Cook EF, Johnson PA, Brand DA, Rouan GW, Lee TH. Prediction of the need for intensive care in patients who come to the emergency departments with acute chest pain. N Engl J Med. 1996;334(23):1498-504.

7. Lee TH, Pearson SD, Johnson PA, Garcia TB, Weisberg MC, Guadagnoli $E$, et al. Failure of information as an intervention to modify clinical management. A time-series trial in patients with acute chest pain. Ann Intern Med. 1995;122(6):434-7.

8. Selker HP, Beshansky JR, Griffith JL, Aufderheide TP, Ballin DS, Bernard SA, et al. Use of the acute cardiac ischemia timeinsensitive predictive instrument (ACI-TIPI) to assist with triage of patients with chest pain or other symptoms suggestive of acute cardiac ischemia. A multicenter, controlled clinical trial. Ann Intern Med. 1998;129(11):845-55.

9. Than M, Cullen L, Reid CM, Lim SH, Aldous S, Ardagh MW, et al. A 2-h diagnostic protocol to assess patients with chest pain symptoms in the Asia-Pacific region (ASPECT): a prospective observational validation study. Lancet. 2011;377(9771):1077-84.

10. Cooper A, Timmis A, Skinner J. Assessment of recent onset chest pain or discomfort of suspected cardiac origin: summary of NICE guidance. BMJ. 2010;340:c1118.

11. Antman EM, Cohen M, Bernink PJ, McCabe CH, Horacek T, Papuchis G, et al. The TIMI risk score for unstable angina/non-ST elevation Ml: A method for prognostication and therapeutic decision making. JAMA. 2000;284(7):835-42.

12. Söderholm M, Deligani MM, Choudhary M, Björk J, Ekelund U. Ability of risk scores to predict a low complication risk in patients admitted for suspected acute coronary syndrome. Emerg Med J. 2012;29(8):644-9.

13. Pope JH, Aufderheide TP, Ruthazer R, Woolard RH, Feldman JA, Beshansky JR, et al. Missed diagnoses of acute cardiac ischemia in the emergency department. N Engl J Med. 2000;342(16):1163-70.

14. Chase M, Robey JL, Zogby KE, Sease KL, Shofer FS, Hollander JE. Prospective validation of the thrombolysis in myocardial infarction risk score in the emergency department chest pain population. Ann Emerg Med. 2006;48(3):252-9.

15. Hess EP, Agarwal D, Chandra S, Murad MH, Erwin PJ, Hollander JE, et al. Diagnostic accuracy of the TIMI risk score in patients 
with chest pain in the emergency department: a meta-analysis. CMAJ. 2010;182(10):1039-44.

16. Hess EP, Perry JJ, Calder LA, Thiruganasambandamoorthy V, Body R, Jaffe A, et al. Prospective validation of a modified thrombolysis in myocardial infarction risk score in emergency department patients with chest pain and possible acute coronary syndrome. Acad Emerg Med. 2010;17(4): 368-75.

17. Amsterdam EA, Kirk JD, Diercks DB, Lewis WR, Turnipseed SD. Immediate exercise testing to evaluate low-risk patients presenting to the emergency department with chest pain. J Am Coll Cardiol. 2002;40(2):251-6.

18. Sprockel JJ, Diaztagle JJ, Chaves WG, Simon CJ, Hernández JI. Estructuración e implementación de una ruta crítica para el manejo de pacientes con síndrome coronario agudo en el Servicio de Urgencias del Hospital San José de Bogotá. Rev Colomb Cardiol. 2013;20(5):262-74.

19. Cox J, Naylor CD. The Canadian Cardiovascular Society grading scale for angina pectoris: is it time for refinements? Ann Intern Med. 1992;117(8):677-83.

20. Resolución 8430 de 1993 Bogotá: Ministerio de Protección Social; 1993.

21. Schuklenk U. Helsinki Declaration revisions. Issues Med Ethics. 2001;9(1):29.

22. Lee TH, Cook EF, Weisberg M, Sargent RK, Wilson C, Goldman L. Acute chest pain in the emergency room. Identification and examination of low-risk patients. Arch Intern Med. 1985;145(1):65-9.

23. Lee TH, Goldman L. Evaluation of the patient with acute chest pain. N Engl J Med. 2000;342(16):1187-95.

24. Arnold J, Goodacre S, Morris F, Team ER. Structure, process and outcomes of chest pain units established in the ESCAPE trial. Emerg Med J. 2007;24(7):462-6.

25. Hamm CW, Bassand JP, Agewall S, Bax J, Boersma E, Bueno H, et al. ESC Guidelines for the management of acute coronary syndromes in patients presenting without persistent ST-segment elevation: The Task Force for the management of acute coronary syndromes (ACS) in patients presenting without persistent ST-segment elevation of the European Society of Cardiology (ESC). Eur Heart J. 2011.

26. Gaspoz JM, Lee TH, Weinstein MC, Cook EF, Goldman P, Komaroff $\mathrm{AL}$, et al. Cost-effectiveness of a new short-stay unit to rule out acute myocardial infarction in low risk patients. J Am Coll Cardiol. 1994;24(5):1249-59.

27. Goodacre SW, Morris FM, Campbell S, Arnold J, Angelini K. A prospective, observational study of a chest pain observation unit in a British hospital. Emerg Med J. 2002;19(2):117-21.

28. Cotarlan V, Ho D, Pineda J, Qureshi A, Shirani J. Impact of clinical predictors and routine coronary artery disease testing on outcome of patients admitted to chest pain decision unit. Clinical cardiology. 2014;37(3):146-51.

29. Hess EP, Nestler DM. Transforming the emergency department observation unit: a look into the future. Cardiology clinics. 2012;30(4):501-21.

30. Richards D, Meshkat N, Chu J, Eva K, Worster A. Emergency department patient compliance with follow-up for outpatient exercise stress testing: a randomized controlled trial. Cjem. 2007;9(6):435-40.

31. Furtado MV, Cardoso A, Patricio MC, Rossini AP, Campani RB, Meotti C, et al. Influence of implementation of a chest pain unit on acute coronary syndrome outcomes. J Emerg Med. 2011;40(5):557-64.

32. Mendoza F. Dolor torácico con sospecha de síndrome coronario agudo; la importancia del juicio clínico, los exámenes, las unidades de dolor torácico y las rutas críticas. Rev Colomb Cardiol. 2013;20(5):275-7.

33. Goldstein JA, Chinnaiyan KM, Abidov A, Achenbach S, Berman DS, Hayes SW, et al. The CT-STAT (Coronary Computed Tomographic Angiography for Systematic Triage of Acute Chest Pain Patients to Treatment) trial. J Am Coll Cardiol. 2011;58(14):1414-22.

34. Hoffmann U, Truong QA, Schoenfeld DA, Chou ET, Woodard PK, Nagurney JT, et al. Coronary CT angiography versus standard evaluation in acute chest pain. $\mathrm{N}$ Engl J Med. 2012;367(4):299-308.

35. Hoffmann U, Truong QA, Fleg JL, Goehler A, Gazelle S, Wiviott $S$, et al. Design of the rule out myocardial ischemia/infarction using computer assisted tomography: a multicenter randomized comparative effectiveness trial of cardiac computed tomography versus alternative triage strategies in patients with acute chest pain in the emergency department. Am Heart J. 2012;163:330-8, 338 e331.

36. Redberg RF. CoronaryCT angiography for acute chest pain. N Engl J Med. 2012;367(4):375-6. 